TRANSACTIONS OF THE

AMERICAN MATHEMATICAL SOCIETY

Volume 190,1974

\title{
REGULAR SELF-INJECTIVE RINGS WITH A POLYNOMIAL IDENTITY
}

BY

\author{
EFRAIM P. ARMENDARIZ AND STUART A. STEINBERG
}

\begin{abstract}
This paper studies maximal quotient rings of semiprime P. I.rings; such rings are regular, self-injective and satisfy a polynomial identity. We show that the center of a regular self-injective ring is regular self-injective; this enables us to establish that the center of the maximal quotient ring of a semiprime P. I.-ring $R$ is the maximal quotient ring of the center of $R$, as well as some other relationships. We give two decompositions of a regular self-injective ring with a polynomial identity which enable us to show that such rings are biregular and are finitely generated projective modules over their center.
\end{abstract}

1. Introduction. E. Formanek's recent result [9] on the existence of central polynomials has been used by $L$. Rowen to show that in a semiprime ring with a polynomial identity each nonzero ideal has nonzero intersection with the center of the ring [17]. Earlier J. Fisher had established that both singular ideals of a semiprime ring with polynomial identity are zero [7]. Subsequently, W. Martindale [13] used Rowen's result to settle two conjectures of Fisher concerning semiprime P. I.rings: (a) the maximal left and right quotient rings coincide; (b) the maximal (left) quotient ring satisfies the same polynomial identity. Since the maximal quotient ring of a ring with zero singular ideal is von Neumann regular and self-injective, this paper investigates the structure of regular self-injective rings with a polynomial identity.

In $\$ 2$ we show that the center of a regular self-injective ring is a regular selfinjective ring; hence the maximal quotient ring of the center of a semiprime P. I.ring is the center of the maximal quotient ring. While a semiprime P. I.-ring with regular center need not be regular we do show that it must be an l-ring and contain an essential regular ideal. $\$ 3$ contains two structure theorems for regular self-injective rings with a polynomial identity; as consequences of our structure theorems we get that such rings are biregular, and, as in the primitive P. I. case, they are finitely-generated (projective) modules over their center. Hence a semiprime P. I.ring can be embedded in a matrix ring over the maximal quotient ring of its center.

Presented to the Society, January 11, 1973 under the title On semiprime P. I. rings and their maximal rings of quotients; received by the editors June 14, 1973.

AMS (MOS) subject classifications (1970). Primary 16A30, 16A38, 16A48; Secondary 16A12, 16A52:

Key words and phrases. von Neumann regular, semiprime ring, polynomial identity, maximal quotient ring, center of a ring. 
Throughout this paper we assume that rings are algebras over commutative rings and that the polynomial identities that occur have at least one invertible coefficient. We denote the maximal left quotient ring of a ring $R$ by $Q$. For the definition and basic properties of quotient rings we refer the reader to [21], [6] and [11]. We note here the following facts:

(a) If $q \in Q$ there is an essential left ideal $D$ of $R$ such that $D q \subseteq R$.

(b) If $R$ has zero (left) singular ideal then a left ideal $D$ of $R$ is essential if and only if the left quotient ring of $D$ is $Q$.

(c) The center of $R$ is contained in the center of $Q$.

(d) If $I$ is an ideal of a semiprime ring $R$ then the center of $I \subseteq$ center of $R$.

We wish to acknowledge many conversations with Joe Fisher and Harvey Wolff which helped in the development of this paper. The referee has also provided many comments which have improved the exposition; we acknowledge his contributions also, especially his observations dealing with Theorem 3.5.

2. The center of a regular injective ring. For the principal result of this section we use Baer's criterion for injectivity [6, p. 5]: A unital left $R$-module $M$ is injective if and only if for each (essential) left ideal $l$ of $R$ and each $R$-homomorphism $f: I \rightarrow M$ there exists $x \in M$ such that $f(a)=a x$ for all $a \in I$. The ring $R$ is (left) self-injective if ${ }_{R} R$ is an injective module.

Theorem 2.1. Let $R$ be a regular left self-injective ring with center $A$. Then $A$ is a regular self-injective ring and $R$ is an injective left (or right) A-module.

Proof. That $A$ is regular is due to J. von Neumann [24] and is well known. Suppose that $I$ is an essential ideal of $A$, and let $f: I \rightarrow A$ be an $A$-homomorphism. Define $f^{*}: R I \rightarrow R$ by setting $f^{*}\left(\Sigma_{j=1}^{n} r_{j} u_{j}\right)=\sum_{j=1}^{n} r_{j} f\left(u_{j}\right)$. Note that $f^{*}$ is well defined. Indeed, if $\sum_{j=1}^{n} r_{j} u_{j}=0$, then $A u_{1}+A u_{2}+\cdots+A u_{n}=A e \subseteq I$ with $e^{2}=$ $e \in A$ as $A$ is regular. Hence $u_{j} e=u_{j}$ for all $1 \leq j \leq n$ and so

$$
\sum_{j=1}^{n} r_{j} f\left(u_{j}\right)=\sum_{j=1}^{n} r_{j} f\left(u_{j} e\right)=\left(\sum_{i=1}^{n} r_{j} u_{j}\right) f(e)=0 .
$$

Thus $f^{*}$ is an $R$-homomorphism; since $R$ is self-injective there exists $r \in R$ for which $f^{*}(t)=\operatorname{tr}$ for all $t \in R I$. We claim that $r \in A$. To see this first note that for $u \in I, u r=f^{*}(u)=f(u) \in A$; hence for all $x \in R$ and $u \in I$ we have $u(x r-r x)=$ $(u x)_{r}-(u r) x=(x u)_{r}-x(u r)=0$, and so $x r-r x \in \mathrm{Ann}_{R} I$. Since $R$ is regular (and hence semiprime), the sum $R I+\mathrm{Ann}_{R}(I)$ is direct. Applying Baer's criterion for injectivity to the map $R I \oplus \mathrm{Ann}_{R}(I) \rightarrow R$ which sends $x+y \rightarrow y$ yields an idempotent $e \in R$ such that $A \mathrm{nn}_{R}(I)=R e$. But $R e$ is an ideal of $R$, since $I$ is a central subset, and in any semiprime ring $R$ an ideal that is a summand of ${ }_{R} R$ is an ideal summand. So $R e$ is unital and hence $e \in A$. Since $I$ was assumed to be 
essential in $A, l e=0$ yields $e=0$. But then $x r-r x=0$ for all $x \in R$ so that $r \in A$ as claimed. A similar proof establishes that $R$ is an injective $A$-module.

Slightly more can be concluded from the hypothesis: $R$ is a torsionless nonsingular $A$-module and by $\mathrm{F}$. Sandomierski [20, Theorem 2.7] if $R$ is a finitelygenerated $A$-module then $R$ is projective.

We remark that the proof shows that if $R$ is a unital self-injective ring with regular center $A$, then $R$ is an injective $A$-module. The question arises: Must $R$ be regular? We have not been able to settle this although it appears unlikely.

Rowen's result mentioned in the introduction shows that the center of a semiprime P. I.-ring is quite large. The next two lemmas give a further indication of the largeness of the center and lead to a generalization of the fact that a semiprime P. I.-ring is simple (prime) if its center is a field (domain).

Lemma 2.2. Let $R$ be a semiprime P. I.-ring with center $A$ and let $D$ be a left ideal of $R$ and $C$ an ideal of $A$. Then (a) $C$ is essential in $A$ if and only if $R C$ is essential in $R$; (b) $D$ is essential in $R$ if and only if $D \cap A$ (= center of $D)$ is essential in $A$.

Proof. (a) If $C$ is essential in $A$, let $J=A \mathrm{nn}_{R}(R C)$. Then for $C_{1}=J \cap A$ we have $C C_{1}=0$ so $C_{1}=0$. By Rowen's theorem [17, Theorem 2], $J=0$ so $R C$ is essential in $R$. Conversely, if $R C$ is essential in $R$ then $\operatorname{Ann}_{A}(C)=\operatorname{Ann}_{R}(C) \cap$ $A=\operatorname{Ann}_{R}(R C) \cap A=0$ so $C$ is essential in $A$.

(b) This is Theorem 6 of Martindale [13].

Lemma 2.3. If $R$ is a semiprime $P$. I.-ring with center $A$ and maximal quotient ring $Q$, then $R$ is an essential A-submodule of $Q$.

Proof. First note that $Q$ is a nonsingular $A$-module. For if $q \in Q$ and $C$ is an essential ideal of $A$, then $C q=0$ yields $R C q=0$, and so $q=0$ since $R C$ is an essential ideal of $R$. If $0 \neq q \in Q$ then there is an essential left ideal $D$ of $R$ for which $D q \subseteq R$. By Lemma 2.2(b), $D \cap A$ is essential in $A$ so $0 \neq(D \cap A)_{q} \subseteq A q \cap R$.

Corollary 2.4. A semiprime P. l.-ring $R$ with center $A$ is self-injective if and only if $R$ is an injective A-module.

Proof. If $R$ is $A$-injective then $R=Q$ by Lemma 2.3. The converse follows from Theorem 2.1.

We use the preceding results to relate the center of a semiprime P. I.-ring to the center of its maximal quotient ring.

Theorem 2.5. Let $R$ be a semiprime $P$. I.-ring with maximal quotient ring $Q$ and let $A=$ center of $R, B=$ center of $Q$. Then $B$ is the maximal quotient ring of $A$.

Proof. We first show that $B$ is an essential extension of $A$ as an $A$-module. Note that $A \subseteq B$ : for if $a \in A, q \in Q$ and $I$ is an essential left ideal of $R$ such that 
$I q \subseteq R$ then $x(a q-q a)=(x a) q-(x q) a=(a x) q-a(x q)=0$; so $a q-q a=0$ since $Q$ is a nonsingular $R$-module. If $b \in B$ then $(R: b)=\{r \in R \mid r b \in A\}$ is an ideal of $R$ which is essential in $R$. Thus $(R: b) \cap A$ is an essential ideal of $A$ by Lemma 2.2(b). Now $A_{R n}(b)$ is not essential in $R$ so by Lemma 2.2(a), $A n_{A}(b)=$ $\operatorname{Ann}_{R}(b) \cap A$ is not essential in $A$. It follows that $(R: b) \cap A \nsubseteq \operatorname{Ann}_{A}(b)$ and so there exists $a \in A$ with $0 \neq a b \in A$ as desired. Next we show that $B$ is an injective $A$-module; so let $C$ be an essential ideal of $A, f: C \rightarrow B$ an $A$-homomorphism and define $f^{*}: B C \rightarrow B$ by $f^{*}\left(\sum_{j=1}^{n} b_{j} c_{j}\right)=\sum_{j=1}^{n} b_{j} f\left(c_{j}\right)$. If $\sum_{j=1}^{n} b_{j} c_{j}=0$ choose an essential ideal $D$ of $A$ such that $D b_{j} \subseteq A$ for all $j=1, \cdots, n$. Then for $d \in D$ we have

$$
d\left(\sum_{j=1}^{n} b_{j} f\left(c_{j}\right)\right)=\sum_{j=1}^{n} f\left(d b_{j} c_{j}\right)=f\left(d\left(\sum_{j=1}^{n} b_{j} c_{j}\right)\right)=0
$$

and it follows that $\sum_{j=1}^{n} b_{j} f\left(c_{j}\right)=0$, since $B$ is a nonsingular $A$-module. Thus $f^{*}$ is well defined. By Theorem 2.1, $B$ is an injective $B$-module so by Baer's criterion, there exists $x \in B$ such that $f(c)=f^{*}(c)=c x$ for all $c \in C$, and so $B$ is an injective $A$-module. Now by $[6$, p. 69] $B$ is the maximal quotient ring of $A$.

Note that something such as a P. I. is needed in Theorem 2.5; for example let $R$ be a prime ring with trivial center. On the other hand, we remark that most of the results in this section hold for strongly regular rings in place of semiprime P. I.rings.

Because the center $A$ of a semiprime P. I. -ring $R$ is so large one might suspect that many properties of $A$ carry over to $R$, e.g. injectivity or regularity. However if $R$ consists of all sequences with entries from a finite-dimensional central division algebra $D$ which are eventually central, then $A$ is self-injective whereas $R$ is not because if $I$ is the ideal of sequences which are eventually zero, the homomorphism $d: I \rightarrow R$ which sends $\left\{x_{n}\right\} \rightarrow\left\{x_{n} d\right\}$, where $d$ is a fixed noncentral element of $D$, cannot be extended to a homomorphism from $R$ to $R$. Also Example 1 of [8] shows that $R$ need not be regular even if $A$ is: there exist nonregular semiprime P. I.-rings which are $\pi$-regular and so have regular center. But even $\pi$-regularity does not carry over because if $R$ is the ring of all sequences of $2 \times 2$ rational matrices which are eventually of the form $\left(\begin{array}{ll}a & 0 \\ 0 & 0\end{array}\right)$ with $a$ integral, then $R$ has regular center but is not $\pi$-regular. This example is however a semisimple l-ring, i.e., each nonzero left ideal contains a nonzero idempotent, and we will show that this is always the case.

Theorem 2.6. Let $R$ be a semiprime P. I.-ring whose center $A$ is a regular ring. Then $R$ is an l-ring and $R$ contains an essential ideal wbicb is a regular ring.

Proof. Let $I \neq 0$ be a left ideal of $R$ and choose a left ideal $J$ of $R$ which is maximal with respect to $I \cap J=0$. Then $U=I+J$ is an essential left ideal of $R$ 
and by Lemma 2.2(b), $C=A \cap U$ is an essential ideal of $A$ and $C$ is a regular ring. If $\lambda$ is an idempotent of $C$, then $\lambda=x+y$ with $x \in I, y \in J$. Hence $\lambda x=x \lambda=x^{2}+x y$ implies $x y \in I \cap J=0$; so $x y=0$ and similarly, $y x=0$. Thus $\lambda=\lambda^{2}=x^{2}+y^{2}$ so $x=x^{2} \in I$. It remains to show $x \neq 0$ for some idempotent $0 \neq \lambda \in C$. If not, then all idempotents of $C$ are in $J$ and therefore $C \subseteq J$ since $C$ is regular. But then by Lemma $2.2(a), J$ is an essential left ideal of $R$ and this contradicts $I \cap J=0$ with $I \neq 0$. Thus $R$ is an $I$-ring. The existence of an essential regular ideal depends on Proposition 3.4 and so we defer its proof till then.

We conclude this section by considering finiteness conditions on the center. The next result has been obtained independently by L. Rowen [18, Appendix ] and has no doubt been noticed by others.

Theorem 2.7. Let $R$ be a semiprime P. I.-ring with center $A$, and let $Q$ be the maximal quotient ring of $R$ with center $B$. Then $R$ is a (left or right) Goldie ring if and only if $A$ is a Goldie ring. In this case $Q=B R$ is the classical quotient ring of $R$.

Proof. For a ring $K$, let $B(K)$ be the Boolean algebra of central idempotents of $K$ and let $A(K)$ be the Boolean algebra of annihilator ideals of $K$. Then since both $Q$ and $B$ are regular self-injective rings, we have $A(Q) \cong B(Q)=B(B) \cong A(B)$. Thus if $R$ is Goldie then $Q$ is semisimple Artinian by Goldie's theorem (cf. [6, p. 76]) so $B$ is semisimple Artinian and hence $A$ is Goldie as $B$ is the maximal quotient ring of $A$. Conversely, if $A$ is Goldie then $B$ is semisimple Artinian and so since $A(Q) \cong A(B)$ and $A(B)$ is finite, $Q$ is a finite direct sum of prime regular self-injective rings (e.g. [6, p. 115]).

As $Q$ is a $P$. I.-ring, Posner's theorem [1] ensures that $Q$ is semisimple Artinian and so $R$ is Goldie. For the final assertion let $q \in Q$ and $C$ an essential ideal of $A$ for which $C q \subseteq R$ (Lemma 2.3). Since $A$ is Goldie, $C$ has a regular element and so $q \in B R$.

We remark that in general $Q \neq B R$ : Let $R$ consist of all sequences with entries from a finite-dimensional central division algebra which are eventually central. Also in Theorem $2.7 Q$ can be obtained by inverting only central elements, as also noted by Rowen [18, Appendix].

3. Two decompositions. Our first structure theorem is

Theorem 3.1. If $R$ is a regular self-injective ring with a polynomial identity, then $R \cong \Pi_{\lambda \in \Lambda} R_{\lambda}$ where each $R_{\lambda}$ is a matrix ring over a strongly regular selfinjective ring with a polynomial identity.

Proof. We first use a theorem of Utumi [22, Theorem 3.2] to write $R$ as $R=$ $S \oplus T$ where $S$ is a strongly regular ring and each nonzero ideal of $T$ contains a 
nonzero nilpotent element. We can assume that $S=0$; then since $R$ is of bounded index, by Levitzki's theorem [10, p. 239] each nonzero ideal of $R$ contains a nonzero ideal which is a matrix ring over a strongly regular ring with unit (such ideals are called matrix ideals in [12]). Any matrix ideal, having a unit, is a direct summand of $R$ and so is itself a regular self-injective ring. In addition, by [23, Theorem 8.3] the strongly regular rings occurring are self-injective. Thus select a maximal independent family $\left\{R_{\lambda} \mid \lambda \in \Lambda\right\}$ of matrix ideals and let $V=\bigoplus \Sigma_{\lambda \in \Lambda} R_{\lambda}$. Since $\operatorname{Ann}_{R}(V)=0, V$ is an essential ideal of $R$ and hence by $[21,2.1], R=Q=$ $Q(V)=\Pi_{\lambda \in \Delta} Q\left(R_{\lambda}\right)=\Pi_{\lambda \in \mathbf{\Lambda}} R_{\lambda}$.

As noted in Armendariz-Fisher [2, Remark 2] every biregular P. I.-ring is a regular ring; however Example 4 of Fisher-Snider [8] shows that the converse does not hold. R. Snider has asked if a self-injective regular P. I.-ring is biregular. Theorem 3.1 provides an affirmative answer. We state this as

Corollary 3.2. A regular self-injective ring $R$ with a polynomial identity is biregular.

Proof. Strongly regular rings are biregular and matrix rings over biregular rings are biregular [12, Lemma 5.2].

Note that the proof of Theorem 3.1 requires only that $R$ is regular left selfinjective and of bounded index for the conclusion to be valid. Since strongly regular left self-injective rings are right self-injective (this can be shown by using [22, Theorem 3.3] or by verifying that if $R$ is such a ring then ${ }_{R} R$ is essential in the maximal right quotient ring of $R$ ) we have the following result of Y. Utumi [21, Theorem 5(1)]:

Corollary 3.3. A regular left self-injective ring of bounded index is right self-injective.

By choosing a different collection of ideals we can obtain a second decomposition of a regular self-injective P. I.-ring. Following A. W.'Chatters [4] we call a P. I.-ring $R$ stable (of degree $n$ ) if $R$ satisfies a polynomial identity of degree $n$ and no nonzero factor of $R$ satisfies a polynomial identity of degree $<n$; an ideal $I$ of $R$ is stable if $I$ is stable as a ring. We can modify a proof of Fisher-Snider [8, Theorem 2.8] to establish

Proposition 3.4. Let $R$ be a semiprime l-ring satisfying a polynomial identity. Then each nonzero ideal of $R$ contains a nonzero (unital) stable ideal.

Proof. Let $K \neq 0$ be an ideal of $R$. If $n$ is the degree of a P. I. of minimal degree satisfied by $K$ set $U=\bigcap\{P \mid P$ is a prime ideal of $K$ and $K / P$ satisfies a $P$. I. of degree $<n\}$. The choice of $n$ guarantees that $U \neq 0$. Moreover any prime ideal of $K$ is an ideal of $R$ : let $P$ be a prime ideal of $K$, then $R P \subseteq K$ and 
$(R P)^{3} \subseteq P$ so $R P \subseteq P$ and similarly $P R \subseteq P$. Thus $U$ is an ideal of $R$. If $Q$ is a proper prime ideal of $U$ then it is easily checked that $P=\{a \in K \mid a U \subseteq Q\}$ is a prime ideal of $K$ and $P \cap U=Q$. As $U / Q \cong(U+P) / P \neq 0, K / P$ satisfies any identity satisfied by $U / Q[1$, Theorem 7$]$ and so by its construction $U / Q$ satisfies no P. I. of degree < $n$ for any proper prime ideal $Q$ of $U$. Again using Levitzki's theorem, $U$ contains a matrix ideal $I$ and as above no prime factor ring satisfies a P. I. of degree < $n$. Since $I$ has a unit, no nonzero factor ring of $I$ satisfies a P. I. of degree $<n$ and so $I$ is stable of degree $n$.

Theorem 3.5. Let $R$ be a regular self-injective ring with a polynomial identity. Then $R=R_{1} \oplus \cdots \oplus R_{k}$ where each $R_{i}$ is eitber zero or else eacb $R_{i}$ is a product of regular self-injective rings each of which is stable of degree $i, 1 \leq i \leq k$.

Proof. Proposition 3.4 enables us to select a maximal-independent family of stable matrix ideals which we can collect according to degree. As in Theorem 3.1 the conclusion now follows.

C. Procesi's version [15] of M. Artin's theorem [3] says that stable unital semiprime P. I.-rings are Azumaya-algebras and as such there is a 1-1 correspondence between the ideals of such a ring and the ideals of its center [5, Corollary 3.7, p. 54]. Using these facts together withProposition 3.4 enables us to complete the proof of Theorem 2.6 that a semiprime P. I.-ring $R$ with regular center $A$ has an essential regular ideal. Since $R$ is an $I$-ring, by Proposition 3.4, it suffices to show that each unital stable ideal $V$ of $R$ is a regular ring. Now $V \cap A=$ center $V$ is an ideal of $A$ and so is a regular ring. Since $V$ is an Azumaya algebra, there is a 1-1 correspondence between the ideals of $V$ and the ideals of $V \cap A$ given in [5, Corollary 3.7, p. 54]; and since prime ideals of $V \cap A$ are maximal ideals, each prime ideal of $V$ is a maximal ideal and hence by [8, Theorem 2.7] $V$ is a regular ring.

The conclusion of Theorem 3.5 can also be rephrased in terms of Azumaya algebras. Let $R$ be a P. I.-ring with unit which satisfies the identities of $n \times n$ matrices over $Z$ but not of $(n-1) \times(n-1)$ matrices and let $F_{n}$ denote Formanek's central polynomial. If $F_{n}(R)$ denotes the set of values taken on by $F_{n}$ then, as noted by Procesi [16, Theorem 3.7] (also Rowen [19, Lemma 9]), $R$ is stable of degree $n$ if and only if $R \cdot F_{n}(R)=R$. It follows that if $\left\{R_{\lambda} \mid \lambda \in \Lambda\right\}$ is a collection of semiprime P. I.-rings with 1 and each $R_{\lambda}$ is stable of degree $n$, with $n$ fixed, then $\Pi_{\lambda \in \Lambda} R_{\lambda}$ is stable of degree $n$. Hence Theorem 3.5 can be stated as follows: If $R$ is a regular self-injective ring with a polynomial identity then $R=R_{1} \oplus \ldots$ $\oplus R_{k}$ where each $R_{i}$ is zero or else each $R_{i}$ is an Azumaya algebra. Thus we have

Corollary 3.6. Let $R$ be a regular self-injective ring with a polynomial identity and center $A$. Then there is a 1-1 correspondence between the ideals of $R$ and 
the ideals of $A$ given by $I \rightarrow I \cap A, U \rightarrow R U$ for $I$ an ideal of $R, U$ an ideal of $A$.

Proof. The correspondence is 1-1 for Azumaya algebras [5, Corollary 3.7, p. 54] and this is extendible to direct sums.

There is another important consequence of Theorem 3.5 when combined with the theory of Azumaya algebras.

Theorem 3.7. Let $R$ be a regular self-injective P. l.-ring with center A. Then $R$ is a finitely-generated projective A-module.

Proof. From Theorem 3.5, $R=R_{1} \oplus \cdots \oplus R_{k}$ where each $R_{i}$ is an Azumaya algebra. Now $A=A_{1} \oplus \ldots \oplus A_{k}$ where $A_{i}=$ center $\left(R_{i}\right)$. As each nonzero $R_{i}$ is an Azumaya algebra, each $R_{i}$ is a finitely-generated projective $A_{i}$-module [5, Theorem 3.4, p. 52]. Thus $R$ is a finitely generated projective $A$-module.

Corollary 3.8. Let $R$ be a semiprime P. I.-ring with center $A$ and let $B$ be the maximal quotient ring of $A$. Then $R$ can be embedded in $B_{n}$, the $n \times n$ matrix ring over $B$, for some $n \geq 1$.

Proof. $R$ embeds in $\operatorname{Hom}_{B}(Q, Q)$ where $Q$ is the maximal quotient ring of $R$. As $Q$ is a finitely-generated projective $B$-module we have $\operatorname{Hom}_{B}(Q, Q) \cong e B_{n} e$ $\subseteq B_{n}$ for some idempotent $e \in B_{n}$.

We conclude by mentioning that the referee has pointed out that the rings $R_{i}$ in the conclusion of Theorem 3.5 can be described as follows: $R_{k}=R \cdot F_{k}(R)$, where $k$ is the largest integer such that $F_{k}(R) \neq 0 ; R_{k-1}=S_{k-1} F_{k-1}(R)$, where $S_{k-1}=\operatorname{Ann}\left(R_{k}\right) ; R_{k-2}=S_{k-2} F_{k-2}(R)$, where $S_{k-2}=\operatorname{Ann}\left(R_{k}+R_{k-1}\right)$; etc.

Added in proof. A more elementary proof of Corollary 3.6 can be given if one notes that the conclusion of the corollary is valid for any biregular ring $R$. Now use Corollary 3.2.

\section{REFERENCES}

1. S. A. Amitsur, Prime rings having polynomial identities with arbitrary coefficients, Proc. London Math. Soc. (3) 17 (1967), 470-486. MR 36 \#209.

2. E. P. Armendariz and J. W. Fisher, Regular P. I.rings, Proc. Amer. Math. Soc. 39 (1973), 247-251.

3. M. Artin, On Azumaya algebras and finite-dimensional representations of rings, J. Algebra 11 (1969), 532-563. MR 39 \#4217.

4. A. W. Chatters, Localization in PI-rings, J. London Math. Soc. (2) 2 (1970), 763-768. MR 42 \#4588.

5. F. DeMeyer and $\mathrm{E}$. Ingraham, Separable algebras over commutative rings, Lecture Notes in Math., vol. 181, Springer-Verlag, Berlin and New York, 1971. MR 43 \#6199.

6. C. Faith, Lectures on injective modules and quotient rings, Lecture Notes in Math. no. 49, Springer-Verlag, Berlin and New York, 1967. MR 37 \#2791. 
7. J. W. Fisher, Structure of semiprime P. I.-rings, Proc. Amer. Math. Soc. 39 (1973), $465-467$.

8. J. W. Fisher and R. L. Snider, On the von Neumann regularity of rings with regular prime factor rings, Pacific J. Math. (to appear).

9. E. Formanek, Central polynomials for matrix rings, J. Algebra 23 (1972), 129-132. MR 46 \#1833.

10. N. Jacobson, Structure of rings, 2nd ed., Amer. Math. Soc. Colloq. Publ., vol. 37, Amer. Math. Soc., Providence, R. I., 1964. MR 36 \#5158.

11. J. Lambek, Lectures on rings and modules, Blaisdell, Waltham, Mass., 1966. MR 34 \#5857.

12. J. Levitzki, On the structure of algebraic algebras and related rings, Trans. Amer. Math. Soc. 74 (1953), 384-409. MR 14, 720.

13. W. S. Martindale III, On semiprime P.I.-rings, Proc. Amer. Math. Soc. 40 (1973), 364-369.

14. E. C. Posner, Prime rings satisfying a polynomial identity, Proc. Amer. Math. Soc. 11 (1960), 180-183. MR 22 \#2626.

15. C. Procesi, On a theorem of M. Artin, J. Algebra 22 (1972), 309-315. MR 46 \#1825.

16. C Central polynomials and finite-dimensional representations of rings (to appear).

17. L. H. Rowen, Some results on the center of a ring with polynomial identity, Bull. Amer. Math. Soc. 79 (1973), 219-223.

18. - On classical quotients of polynomial identity rings with involution, Proc. Amer. Math. Soc. 40 (1973), 23-29.

19. - On rings with central polynomials, J. Algebra (to appear).

20. F. L. Sandomierski, Nonsingular rings, Proc. Amer. Math. Soc. 19 (1968), 225230. MR 36 \#2648.

21. Y. Utumi, On quotient rings, Osaka Math. J. 8 (1956), 1-18. MR $18,7$.

22. - On rings in which any one-sided quotient rings are two-sided, Proc. Amer. Math. Soc. 14 (1963), 141-147. MR 26 \#137.

23. - On continuous rings and self-injective rings, Trans. Amer. Math. Soc. 118 (1965), 158-173. MR 30 \#4793.

24. J. von Neumann, Regular rings, Proc. Nat. Acad. Sci. U. S. A. 22 (1936), 707-713.

DEPARTMENT OF MATHEMATICS, UNIVERSITY OF TEXAS, AUSTIN, TEXAS 78712

DEPARTMENT OF MATHEMATICS, UNIVERSITY OF TOLEDO, TOLEDO, OHIO 43606 